\title{
Calidad en Producción de Maquetas y Tutoriales en Línea
} Scale Models Quality Production and Online Tutorials

\author{
Mario Yadir Rendón Sallard \\ Universidad de Sonora, México \\ yadir42@hotmail.com
}

\author{
Rosa María Mendoza Robles \\ Universidad de Sonora, México \\ rosa.mendoza@unison.mx
}

\author{
Fernando Saldaña Córdoba \\ Universidad de Sonora, México \\ fsaldana@arq.uson.mx
}

\begin{abstract}
The university student faces multiple challenges to acquiring knowledge for their degrees. We study the ways the architecture student at Universidad de Sonora overcomes such difficulties, in a process of academic resilience, through the use of a video tutorial repository covering topics related to architecture scale model fabrication, among other study materials. This repository meets the demand for web-based, Spanish-language study materials. It was tested in terms of usefulness and acceptability: video tutorials were not only considered helpful by the students, but a handy tool when attempting to learn new abilities and knowledge, or reinforce those they already acquired.
\end{abstract}

Keywords: Maquetas; Tutoriales; Arquitectura; Educación Resiliente

\section{Introducción}

La formación de recursos humanos universitarios requiere el uso de técnicas de enseñanza innovadoras que respondan a la evolución social y de mercado y se adapten a las necesidades de los estudiantes. En el caso de muchas de las universidades mexicanas el esquema curricular del estudiante de arquitectura no incluye la fabricación de maquetas de ningún tipo; sin embargo en gran cantidad de las clases teóricas y de taller de diseño se solicitan maquetas de estudio y de presentación.

Ante la necesidad de proveer a los estudiantes con material de apoyo accesible, pedagógicamente relevante, atractivo, y en su lengua materna se desarrolló en el Departamento de Arquitectura y Diseño de la Universidad de Sonora, Mexico, un proyecto de tutoriales en video, para apoyar el aprendizaje en técnicas de presentación de proyectos arquitectónicos. Este proyecto se ha realizado desde 2014, en la etapa inicial del mismo se desarrollaron y evaluaron video-tutoriales de diferentes técnicas de expresión gráfica manual (Mendoza, Rendón y Saldaña, 2015). Dichos tutoriales se encuentran accesibles vía web, desde un sitio universitario abierto creado con este fin -http://www.dad.uson.mx/tutoriales-. En esta segunda etapa, se desarrollaron y evaluaron video-tutoriales para la elaboración de maquetas arquitectónicas. Con este estudio esperamos entender los retos que enfrenta el estudiante para lograr una mejor producción de maquetas, adaptar las herramientas a los estudiantes actuales y lograr una educación resiliente.

\section{Justificación}

La resiliencia se define como la capacidad de un sistema para responder a una alteración y reorganizarse ante el cambio para conservar esencialmente la misma función, estructura, identidad y mecanismos de retroalimentación (Walker et al., 2004). El concepto de resiliencia, aplicado a sistemas integrados de personas y ambiente natural por C.S. Holling en 1973, tiene tres características interrelacionadas: 1) la cantidad de cambio que puede soportar un sistema sin perder control sobre sus funciones y estructura, 2) el grado hasta el cual el sistema puede auto-organizarse, y 3) la capacidad del sistema para construir e incrementar el aprendizaje y la adaptación (http://www.resillience.org/). En sistemas resilientes, el cambio y la diversidad no son percibidos como amenazas, sino como condiciones necesarias para la renovación, la actualización y el aprendizaje (Marcus y Colding, 2014).

Aunada a la necesidad de compartir con los estudiantes universitarios de arquitectura los principios y las condiciones necesarias para construir y mantener sistemas ecológicos resilientes, también existe la necesidad de promover la resiliencia académica o educativa para mejorar los procesos de aprendizaje y disminuir la incidencia de abandono escolar. Jowkar et al. (2014) argumentan que la motivación es un factor elemental para la resiliencia académica, e identifican factores protectores internos y externos que la promueven, incluyendo la auto-eficacia en el estudio. Este proyecto busca contribuir a la auto-eficacia al disminuir las deficiencias en las capacidad o habilidades que tienen los estudiantes para enfrentar las demandas del programa educativo, incluyendo conocimiento inadecuado, actitudes inapropiadas hacia el aprendizaje y bajo nivel de resiliencia psicológica, entre otros (Araque, Roldán y Salguero, 2009).

El uso de tutoriales en video fomenta el auto-estudio en alumnos acostumbrados a recibir la información ya procesada. El uso efectivo de video-tutoriales ocurre cuando los mismos están bien diseñados, tienen un mecanismo de evaluación y están fácilmente disponibles (Wells, Barry y Spence, 2012). 
Los videos permiten que el alumno aprenda a su propio ritmo y genere resiliencia en la medida en que se apropia del conocimiento (Wang y Gordon, 1994). Además, proporcionar herramientas alternativas a las que se utilizan en el aula también refuerza el rol del profesor como un facilitador de conocimientos y herramientas (Comiskey y McCartan, 2011; Leijen et al., 2009; Paliokas, 2009).

Específicamente para el programa de arquitectura de la Universidad de Sonora la encuesta aplicada al 30\% del estudiantado -267 de 865 alumnos inscritos- en todos los niveles arrojó lo siguiente:

- El $57 \%$ de los estudiantes considera que las dificultades para lograr el aprendizaje son personales: hambre, falta de sueño, cansancio, etc.

- Cuando lo explicado en clase es insuficiente, el estudiante acude a tres herramientas principalmente, búsquedas en internet, tutoriales en línea y asesoría con pares.

- El alumno evita la asesoría con su profesor principalmente por razones de tiempo -personales o del profesor-.

- La opción más popular para reforzar el conocimiento e información es la búsqueda en internet con un $87 \%$ del estudiantado utilizándolas más de 2 veces por semana.

- Más del $23 \%$ de los alumnos nunca ha visitado la biblioteca, contrasta con más del $56 \%$ de los estudiantes que utilizan tutoriales al menos una vez por semana.

- El motivo de utilización de tutoriales es principalmente académico: 3 de cada 4 veces.

Otros estudios existentes que evalúan el uso de videotutoriales para la educación arquitectónica han documentado un impacto positivo en el aprendizaje porque proporcionan flexibilidad y animan a los estudiantes a ser más activos (Comiskey y McCarten, 2011), asimismo también se documentan algunos inconvenientes, constituyendo el principal de ellos la dificultad para encontrar videos de calidad que se apeguen a la materia en cuestión (Herreid y Schiller, 2013). Esto es particularmente importante para nuestra institución, donde no hay una materia dedicada específicamente para enseñar la elaboración de maquetas arquitectónicas, aunque en muchas materias se requiere que los estudiantes hagan maquetas como parte de los proyectos que estudian y desarrollan. Como apoyo para que el estudiante complemente sus conocimientos y desarrolle resiliencia académica, se han desarrollado video-tutoriales accesibles desde el sitio web del Departamento de Arquitectura y Diseño de la Universidad de Sonora. Aquí presentamos el estudio realizado sobre la modificación en la calidad de producción de maquetas.

\section{Metodología}

Estudiamos la modificación en la calidad de producción de maquetas a partir de dos actividades: corte y pegado. La metodología utilizada consta de las siguientes fases: a) planeación del proyecto, b) recopilación de información existente en videos similares, c) elaboración del guión, d) grabación de tutoriales, e) edición de video, f) pruebas piloto y evaluación de modificación de calidad vía entrevistas y productos, g) correcciones a los videos, h) publicación.

Estos tutoriales se encuentran accesibles vía web, desde el sitio universitario creado con ese fin: (http://www.dad.uson.mx) o en su canal de youtube.

Para las pruebas se invitó a un grupo de 19 participantes (Figura 1), de primer y segundo año de arquitectura, los cuales realizaron dos ejercicios, uno preliminar y otro posterior al video-tutorial. Esto con la finalidad de medir la modificación de nivel de calidad en corte, pegado y limpieza después del video-tutorial. Una vez realizados ambos ejercicios (Figura 2) se aplicó un cuestionario que indagó sobre sus herramientas de aprendizaje, así como sobre la calidad del video en sí.

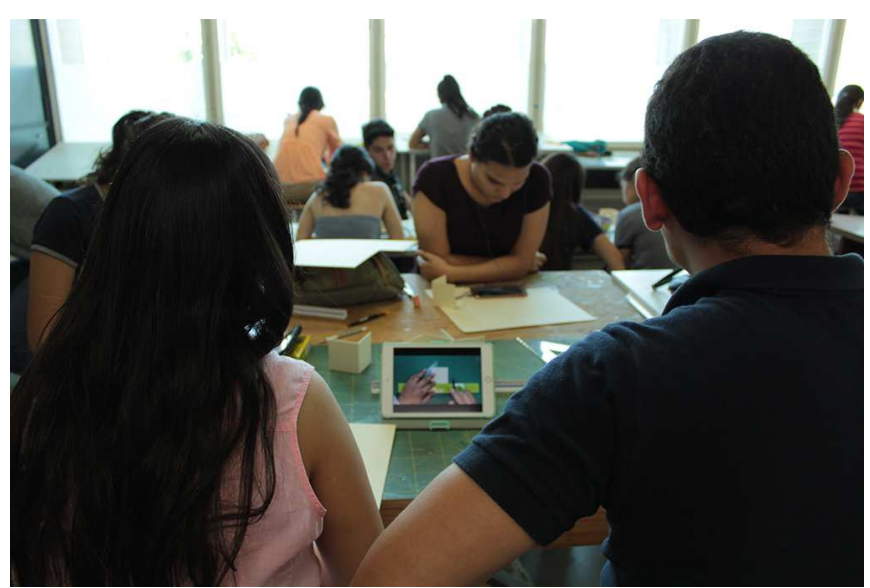

Figura 1: Participantes observando el video-tutorial.

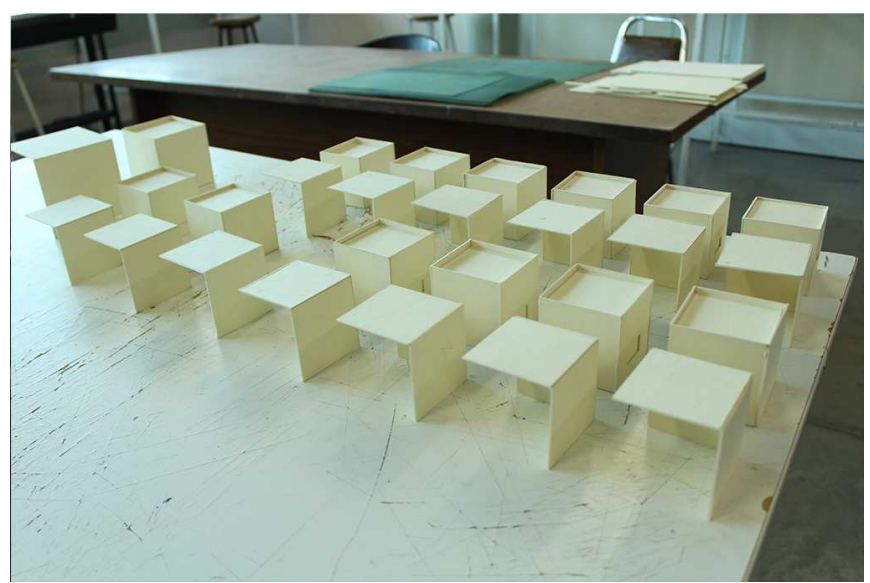

Figura 2: Maquetas realizadas en el ejercicio.

A los participantes se les facilitaron herramientas y material necesario para realizar los ejercicios solicitados. En el ejercicio previo se pidió que realizaran 3 caras de un cubo, cortándolas y pegándolas con los conocimientos que tenían. Posteriormente se les solicitó que realizaran el ejercicio del video siguiendo las instrucciones del mismo. Los participantes terminaron ambos ejercicios en un promedio de 2 horas. Posteriormente se analizaron individualmente las maquetas elaboradas por cada participante, revisando con lente de aumento la calidad de corte, pegado y limpieza; se revisó lo que se aprecia a simple vista. 
Es importante señalar que en los criterios de evaluación se consideró la mejora o deterioro de calidad de cada uno de los aspectos estudiados, más allá de la perfección final del producto, ya que se reconoce que éstos son elaborados por estudiantes sin conocimientos formales de elaboración de maquetas, y como se señaló anteriormente, este rubro no se incluye en el currículo formal de nuestros estudiantes.

\section{Resultados}

Después de la observación minuciosa, y análisis de cada uno de los productos de los participantes se realizó una comparativa de incremento o decremento en calidad de corte pegado y limpieza, la cual arrojó los resultados que comentamos a continuación. Ejemplos de esta comparativa se encuentran en la Figura 3.

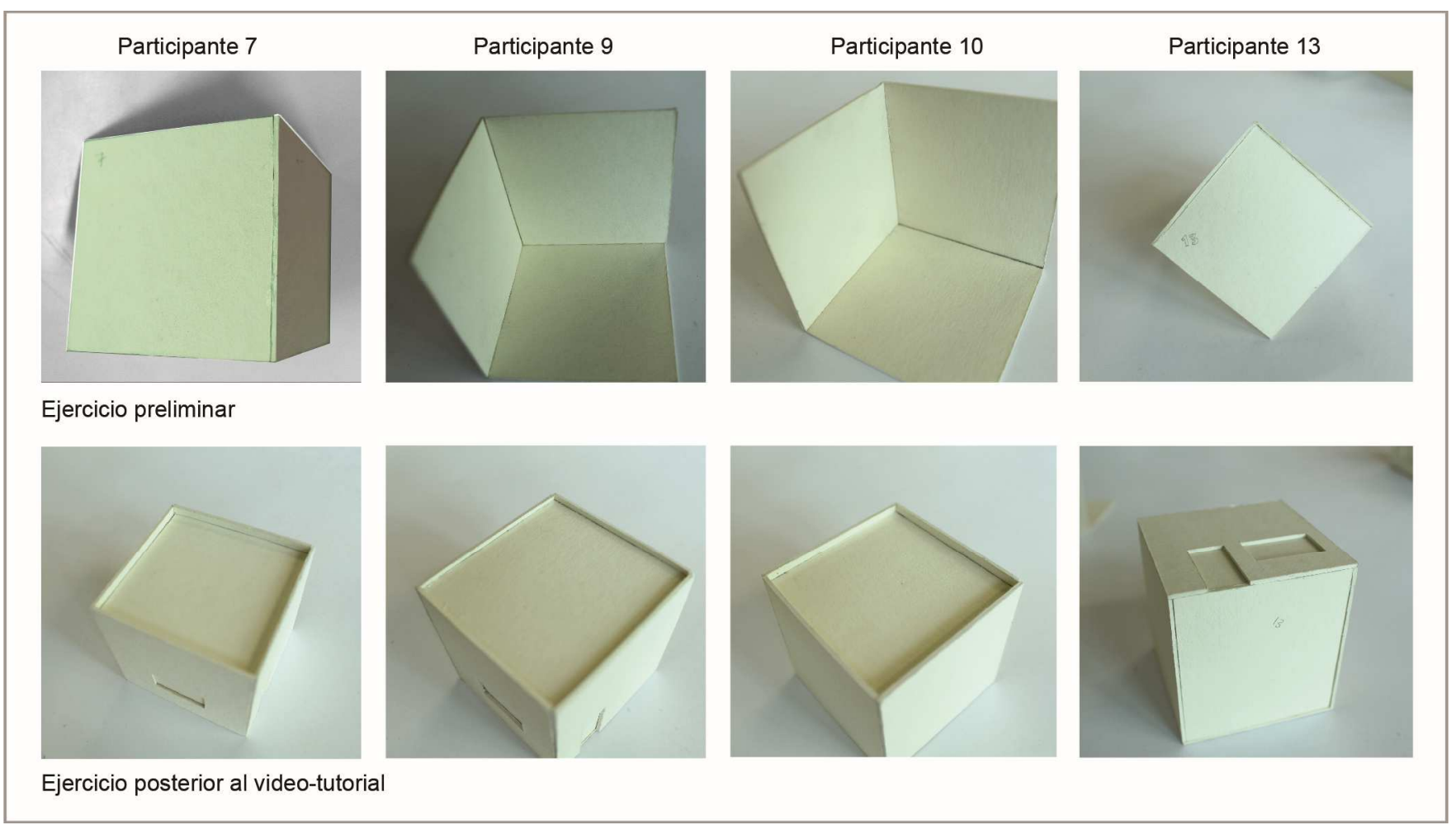

Figura 3: Ejemplos comparativos por alumno del ejercicio previo con el ejercicio posterior a la visualización del tutorial

En el aspecto de corte, cerca de la mitad $-47 \%$ de los participantes- mejoraron la calidad de corte en el segundo ejercicio, de los restantes el $26 \%$ mantuvo el nivel de calidad de corte y el otro $26 \%$ mostró un deterioro en su calidad.

Sólo dieciocho participantes acabaron la segunda maqueta, de los cuales, el 50\% mantuvo el nivel de calidad de pegado similar en ambas maquetas, y el resto mostró una mejora significativa en su calidad de pegado.

En el aspecto de limpieza, arriba de la mitad $-58 \%$ de los estudiantes- mantuvieron el nivel de limpieza similar en ambos ejercicios. Sólo un $17 \%$ de los estudiantes mostró mejora en el nivel de limpieza. Por lo cual es asumible que la visualización del tutorial no tuvo efecto en el aspecto de mejora de limpieza.

En los ejercicios que se observaron decrementos de calidad de los puntos analizados faltará por comprobar en futuros ejercicios si se debió a factores personales —tiempo, hambre, nivel individual de exigencia- o es imputable al propio videotutorial; sin embargo las opiniones en la calidad del tutorial, que se presentan más adelante en este artículo, sugieren que se debe a factores personales.

La aplicación de este ejercicio nos demostró la conveniencia de indicar explícitamente al inicio de cada tutorial señalamientos que consideramos evidentes previamente, como son:

- Revisar que la navaja de corte tenga filo o que sea nueva de preferencia.

- Verificar que los instrumentos como escuadras y reglas estén limpios.

- Preparar el área de trabajo y tener a la mano todas las cosas que se utilizarán en el ejercicio.

- Verificar que el material a utilizar se encuentre limpio, sin marcas ni daños.

- Verificar que las manos estén limpias.

\section{Opiniones de los participantes}

La capacidad del estudiante de enfrentarse a las dificultades de aprendizaje, o resiliencia educativa, es desarrollada con 
apoyos diversos, por lo cual solicitamos a los participantes del ejercicio seleccionar entre diversas opciones: los videotutoriales figuraron como la tercer forma de apoyo a la hora de enfrentar dificultades de aprendizaje en sus estudios; cabe señalar que la más popular fue la consulta de internet en general y en segundo lugar la asesoría con amigos. Más detalles de estos datos se encuentran en la Figura 4, donde también podemos observar que la asesoría con maestros es de las soluciones menos recurridas por los participantes.

Durante el semestre la utilización de video-tutoriales en línea es alta, el $58 \%$ de los participantes utilizan video-tutoriales 4 veces o más en el semestre escolar, y el $42 \%$ señaló utilizar video-tutorales al menos una vez al semestre, ninguno de los participantes marcó la casilla de cero utilización. Sin embargo, detectamos un desconocimiento del recurso institucional que estamos desarrollando: en una muestra de doscientos alumnos sólo el 15\% conocía el sitio. En contraparte, la finalidad de la consulta de video-tutoriales fue señalada como académica principalmente.

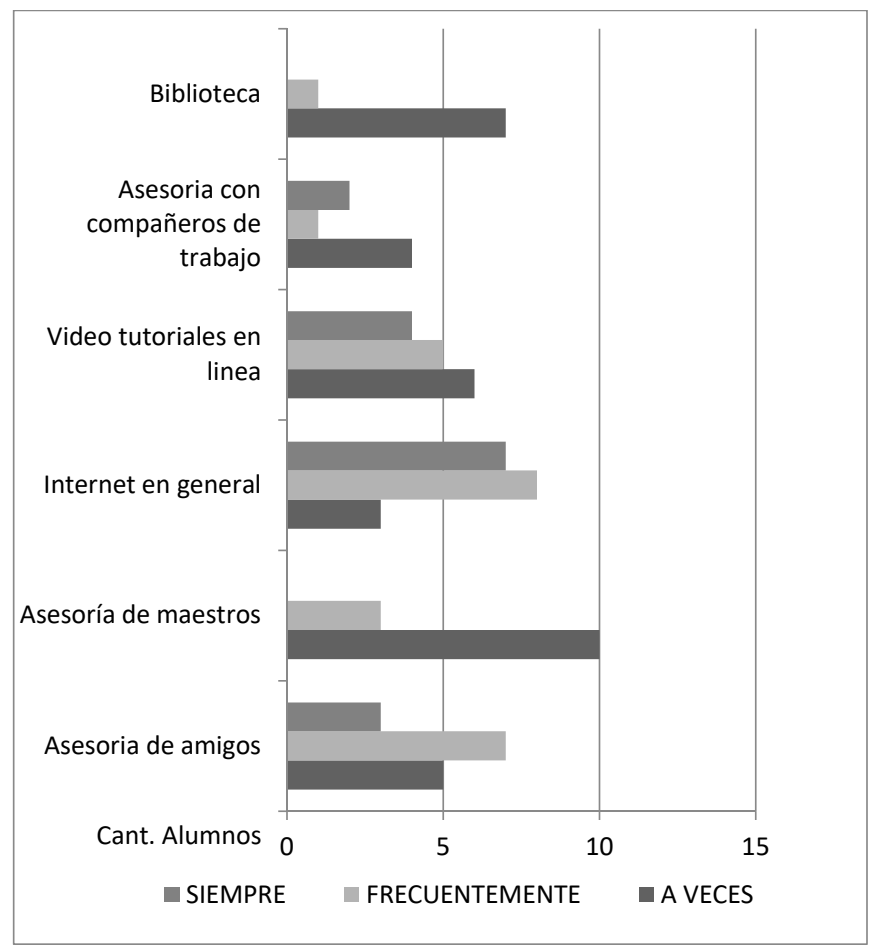

Figura 4: Gráfica de utilización y frecuencia de soluciones de resiliencia educativa.

Las razones principales para la utilización de video-tutoriales como forma de estudio fueron elegidas: la rapidez de consulta, la flexibilidad de ubicación del estudiante para la consulta y la actualidad de la información presentada en los videos. La finalidad de la consulta de video-tutoriales fue señalada como académica principalmente en el sondeo previo que realizamos.

En cuanto a la evaluación del video-tutorial de maquetas que se aplicó los participantes consideraron la información presentada en el video como muy detallada $-84 \%$ de las respuestas-. La calidad de la imagen, del sonido, y explicación fue considerada de buena a muy buena por el $95 \%$ de los paticipantes, sin embargo, encontramos áreas de mejora en las notas sobre la logitud del video, señalando esta última como larga -la duración total de los videos utilizados fue de 22:46 minutos, dividido en tres partes de: 7:47, 7:46 y 6:55 minutos respectivamente-.

Luego de realizar el ejercicio todos los participantes reconocieron una mejora sustancial en su habiliad de uniones, escuadre y metodología de ensamble. Buenos resultados se obtuvieron también en las habilidades de corte y trazo, de diecinueve solo uno de los estudiantes consideró no haber mejorado su habilidad de corte ni de limpieza, y dos de ellos no consideraron mejora en su habilidad de trazo. En el aspecto de calidad, la tendencia de los niveles de mejora personal en todos los rubros fue seleccionada de media a alta.

\section{Conclusión}

Representa un reto para este proyecto y otros similares cumplir con una necesidad creciente de compartir conocimientos en un mundo de múltiples vías de comunicación y redes tecnológicas, y sobre todo para generar herramientas que resulten atractivas para los estudiantes y que ellos mismos demandan, y que además fomenten las relaciones interpersonales, la curiosidad y la estimulación intelectual, que son los componentes necesarios para una enseñanza de calidad, como han identificado Bristow, Bruhl y Klosky (2014).

Compartimos con Monedero (2007) la preocupación por generar recursos que promuevan tanto enseñanzas e instrucciones adecuadas como una formación y educación de orden superior (p. 157-158). Consideramos que el uso de video-tutoriales es una de varias herramientas que fomentan las habilidades metacognitivas del estudiante (Paliokas, 2009) y promueven una mejor satisfacción y mejores resultados de aprendizaje porque fomentan la habilidad para enfrentar retos y persistir con una tarea aun cuando les resulta difícil (Jowkar et al., 2014).

Las pruebas aquí presentadas coinciden en lo encontrado con los estudios anteriores, sin embargo es nuestro deber señalar discrepancia encontrada en los resultados físicos y las opiniones de la encuesta: en el rubro de corte el $26 \%$ de participantes no mostraron en su producto la mejora que señalaron en la encuesta haber adquirido. Este deterioro en la calidad de corte lo atribuimos a razones de confort y prisa de estos estudiantes, ya que las observaciones que nos hicieron en la encuesta son predominantemente positivas en todos los casos, sin embargo los cometarios que se obviaron se han tomado en cuenta para incluir esos señalamientos en todos los videos

Asimismo, la popularidad del sitio de Video Tutoriales Unison, así como la del canal de videos https://www.youtube.com/channel/UCO8mXB0sODZgYdB0Ci i7PKw ha ido en incremento desde que se publicó en septiembre de 2016 y al momento de escribir este artículo cuenta con 14,028 visualizaciones y 314 suscriptores, que muestran que los video-tutoriales son una herramienta útil y atractiva, sobre todo para alumnos que por cuenta propia 
están dispuestos a mejorar su calidad de trabajo o resolver problemas de una educación incompleta o incluso deficiente, construyendo de esta forma resiliencia educativa.

\section{Agradecimientos}

Damos las gracias a la Universidad de Sonora por el apoyo en recursos físicos y económicos para la realización de este proyecto. Agradecemos a todos los participantes del ejercicio de evaluación, a Gabriela Grijalva y Oscar Corella por su colaboración con la edición de los video-tutoriales, así como a Gustavo Salas y José Manuel Ochoa, a El Atelier y a Kwick Papelería por apoyos de equipo y patrocinio de materiales.

\section{Referencias}

Araque, F., Roldán, C., y Salguero, A. (2009). Factors influencing university drop out rates. Computers y Education, 53(3), 563-574. http://dx.doi.org/10.1016/j.compedu.2009.03.013

Bristow, E., Bruhl, J., y Klosky, J. (2014). Effect of supplemental instructional videos on student performance in engineering mechanics class. International Journal of Engineering Education, 30(3), 566-575.

Comiskey, D., y McCartan, K. (2011). Video: An effective teaching aid? An architectural technologist's perspective. CEBE Transactions, 8(1), 16.

Jowkar, B., Kojuri, J., Kohoulat, N., y Hayat, A. (2014). Academic resilience in education: The role of achievement goal orientations. Journal of Advances in Medical Education and Professionalism, 2(1), 33-38.

Herreid, C.F. y Schiller, N.A. (2013). Case studies and the Flipped Classroom. Journal of College Science Teaching, 42(5), pp. 62-
66. http://aacu.org/sites/default/files/files/PKAL_regional/CRWGSPEE-REF-01.pdf

Leijen, A., Lam, I., Wildschut, L., Simons, P. R.-J., y Admiraal, W. (2009). Streaming video to enhance students' reflection in dance education. Computers y Education, 52(1), 169-176.

Marcus, L., y Colding, J. (2014). Toward an integrated theory of spatial morphology and resilient urban systems. Ecology and Society, 19(4), 55. http://dx.doi.org/10.5751/ES-06939-190455

Mendoza Robles, R., Rendón Sallard, M., y Saldaña Córdova, F. (2015). Videotutoriales para representación gráfica. En L. Irigoyen, M. Aguilar Tobin y A. Elizalde García, Agentes dinámicos en la enseñanza del diseño gráfico (pp. 29-40). Hermosillo: Universidad de Sonora, Quartuppi. Recuperado de http://www.qartuppi.com/agentes.pdf

Monedero, J. (2007). Architectural eLearning. Una indagación sobre los límites borrosos que separan la educación y la instrucción. 11 Congreso de la Sociedad Iberoamericana de Gráfica Digital, Cd. De México, México, 23-25 octubre 2007. Recuperado de http://papers.cumincad.org/

Paliokas, I. (2009). Reinforcing Metacognition in CAD Education using Videotutorials. Computer-Aided Design and Applications, 6(5), 11.

Walker, B., Holling, C., Carpenter, S., y Kinzig, A. (2004). Resilience, Adaptability and Transformability in Social-ecological Systems. Ecology And Society, 9(2). http://dx.doi.org/10.5751/es-00650090205

Wang, M. C., y Gordon, E. W. (1994). Educational resilience in innercity America: Challenges and prospects. Hillsdale, N.J.: L. Erlbaum Associates.

Wells, J., Barry, R. M. y Spence, A. (2012). Using Video Tutorials as a Carrot-and-Stick Approach to Learning. IEEE Transactions on Education, 55(4), pp. 453-458. http://dro.deakin.edu.au/eserv/DU:30046990/wells-usingvideopost-2012.pdf 